\title{
Stock Price Prediction using Technical Indicators: A Predictive Model using Optimal Deep Learning
}

\author{
Manish Agrawal, Asif Ullah Khan, Piyush Kumar Shukla
}

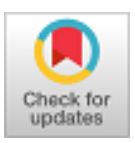

\begin{abstract}
The stock market is highly volatile and complex in nature. Technical analysts often apply Technical Analysis (TA) on historical price data, which is an exhaustive task and might produce incorrect predictions. The machine learning coupled with fundamental and / or Technical Analysis also yields satisfactory results for stock market prediction. In this work an effort is made to predict the price and price trend of stocks by applying optimal Long Short Term Memory (O-LSTM) deep learning and adaptive Stock Technical Indicators (STIs). We also evaluated the model for taking buy-sell decision at the end of day. To optimize the deep learning task we utilized the concept of Correlation-Tensor built with appropriate STIs. The tensor with adaptive indicators is passed to the model for better and accurate prediction. The results are analyzed using popular metrics and compared with two benchmark ML classifiers and a recent classifier based on deep learning. The mean prediction accuracy achieved using proposed model is $59.25 \%$, over number of stocks, which is much higher than benchmark approaches.
\end{abstract}

Keywords: Deep Learning, Data Science, Machine Learning, Long Short Term Memory, National Stock Exchange, Predictive Modelling, Stock Market Prediction, Stock Technical Indicators, Technical Analysis.
Table 1: Nomenclature

\begin{tabular}{ll}
\hline Abbreviations & \multicolumn{1}{c}{ Full Form } \\
\hline API & Application Programming Interface \\
ANN & Artificial Neural Network \\
ARIMA & Auto-Regressive Integrated Moving \\
CNN & Average \\
DNN & Convolutional Neural Network \\
EMA & Deep Neural Network \\
FA & Fundamential Moving Average \\
LR & Logistic Regression \\
LSTM & Long Short Term Memory \\
MACD & Moving Average Convergence \\
ML & Divergence \\
NN & Machine Learning \\
MSE & Neural Network \\
MLP & Mean Squared Error \\
NSE & Multi Layer Perceptron \\
NYSE & National Stock Exchange \\
RNN & New York Stock Exchange \\
RSI & Recurrent Neural Network \\
SBI & Relative Strength Index \\
SMA & State Bank of India \\
STI & Simple Moving Average \\
SVM & Stock Technical Indicators \\
TA & Support Vector Machine \\
\hline & Technical Analysis \\
\hline &
\end{tabular}

\section{INTRODUCTION}

In an attempt to predict stock market trends and future stock prices, market researchers, investors and scholars regularly propose a range of models.

These models are based on various methods including the following.

1. Single and dual source of information [2]. Single information source methods either utilize numerical or semantic information extracted from news and reviews while dual source methods utilize historical price and volume data as well.
Revised Manuscript Received on 30 July 2019.

* Correspondence Author

sManish Agrawal, Phd Scholar Computer Science \& Engineering UIT, RGPV, Bhopal, (M.P.) India.

Dr Asif Ullah Khan, Professor Department of CSE TIT-(E), RGPV Bhopal, (M.P.) India.

Dr Piyush Kumar Shukla, Assistant Professor Department of CSE UIT, RGPV, Bhopal, (M.P.) India.

(C) The Authors. Published by Blue Eyes Intelligence Engineering and Sciences Publication (BEIESP). This is an open access article under the CC-BY-NC-ND license http://creativecommons.org/licenses/by-nc-nd/4.0/

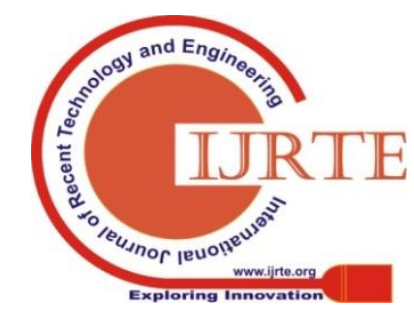




\section{Stock Price Prediction using Technical Indicators: A Predictive Model using Optimal Deep Learning}

2. Fundamental Analysis (FA), Technical Analysis (TA) and Time Series Forecasting [3]. The Fundamental analysis utilizes sales, earnings, profits and other economic factors for forecasting. The TA utilizes some stock indicators like Moving Averages (MAs) for forecasting.

Time series forecasting utilizes linear model like ARIMA $[4,5]$ and Non-Linear models such as ANN and Deep Learning [6, 7].

Most of recent research works employed ML algorithms to predict stock price movement. Two most common ML approaches are ANN [8-10] and Support Vector Machine (SVM) [11, 12]. These are widely used for long and short term analysis and prediction of stock trends. Such methods generally utilize STIs calculated from past prices and volume data. To determine, which STIs, financial news and algorithm is more effective and how to analyze these to increase profits have drawn much interest from the research community. Investigating deep learning (aka Deep Neural Network) [13, 14] techniques in financial time series prediction is a highly recommended solution. E. Chong et al. [15] use only numerical data to explore the effects of deep learning method in stock market prediction. The approach proposed in this work is capable of identifying hidden relationships and underlying dynamics in the historic price data through an optimal LSTM deep learning approach. Originally LSTM is proposed by Hochreiter and Schmidhuber [1]

\section{Problem Statement:}

- The investors usually take the decisions of buying or selling the stock by evaluating a company's performance and other unexpected global, national \& social events. Although, such events eventually affect stock prices instantaneously in a negative or positive way, these effects are not permanent most of the time. So, it is not viable to predict the stock prices and trends on the basis of FA.

As a consequence an automated system or model, to analyse the stock market and upcoming stock trends based on historical prices and STIs, is needed.

- Determining highly correlative STIs based on historical price data is an exhaustive task and might produce incorrect predictions. So, determining highly correlative STIs is another challenge and dealt as a problem in this work.

- Applying traditional machine learning and deep learning approaches yields average results as the stock market follows random walk motion. Applying Optimal LSTM Deep Learning algorithm and adaptive STIs can make an effective forecast. We made an effort to combine the concept and achieved better results.

- Most of the previous works have not evaluated the algorithm for prediction accuracy and suffer from overfitting.

\section{Key Contributions of this Work:}

Based on the problem statement, we present:

- Optimal LSTM Deep Learning Approach to achieve better prediction of stock market trends using Adaptive STIs.

- The concept of Correlation tensor derives appropriate STIs based on historical prices and volume data. The concept of correlation also speeds up the deep learning model.
- Performance evaluation and comparison of proposed work (approach) against popular ML classifiers is presented.

- Higher Prediction Accuracy and less Mean Squared Error.

\section{Outcomes:}

As an outcome the proposed work aids investor in making good investment decisions to earn profits. The framework also gives decision indicator [Price-rise (1) and Price-fall (0)] to the stock holder at the end of each day. The proposed approach can also be used as trend based analysis to decide whether to hold stocks for long or short term.

The rest of this paper is organized as follows. Section 2 presents review of related literature.

In section 3, we discuss the concept of correlation tensor, framework with Optimal LSTM for stock trends and closing price prediction (high/low) for next day. Section 4 presents experimental setup, methodology, results, and performance analysis for the evaluation of the proposed work. In section 5 we conclude with future directions.

\section{LITERATURE REVIEW}

\subsection{Stock Technical Indicators (STIs)}

STIs are statistical calculations based on the price, volume, or significance for a share, security or contract. These does not depends on fundamentals of a business, like earnings, revenue, or profit margins. The active stock traders and technical analysts commonly use STIs to analyze short-term and long term price movements and to identify entry and exit points [16]. Technical indicators $[17,18]$ can be useful while predicting the future prices of assets so they can be integrated into automated trading systems. There are two basic types of technical indicators: Overlays and Oscillators. In this work, we use SMA as it is one among the most widely used STI. It filters out the noise which occurs due to random price variations and helps to smooth out price. It is said as trend following indicator or simply lagging as it depends on past prices.

Formulae for calculating the most prevailing Stock Technical Indicators (STIs) is presented in Table 2.

Table 2: Stock Technical Indicators

\begin{tabular}{|l|c|}
\hline \multicolumn{1}{|c|}{$\begin{array}{c}\text { Stock Technical } \\
\text { Indicators (STIs) }\end{array}$} & Mathematical Formula \\
\hline $\begin{array}{l}\text { Relative Strength } \\
\text { Index (RSI) }\end{array}$ & $R S I=100-\left[\left(\frac{100}{1+\left(\frac{A G}{A L}\right)}\right)\right]$ \\
\hline $\begin{array}{l}\text { Moving Average } \\
\text { (MA) of n days }\end{array}$ & $\frac{1}{n} \sum_{i=1}^{n} C_{i}$ \\
\hline $\begin{array}{l}\text { Stochastic Oscillator } \\
\text { (\%K) }\end{array}$ & $\% K=\left[\frac{C_{t}-L p}{H p-L p}\right] * 100$ \\
\hline William (\%R) & $\% K=\left[\frac{H p-C_{t}}{H p-L p}\right] * 100$ \\
\hline $\begin{array}{l}\text { Exponential Moving } \\
\text { Average (EMA) }\end{array}$ & $=C_{t}\left(\frac{2}{T+1}\right)$ \\
& $+E M A_{t-1}\left(1-\frac{2}{T+1}\right)$ \\
\hline
\end{tabular}




\begin{tabular}{|l|l|}
\hline Moving Average & MACD \\
Convergence & $=[(12-$ day $E M A)-(26$ \\
Divergence (MACD): & $-\operatorname{day} E M A)]$ \\
most common is & \\
12/26 MACD & \\
\hline
\end{tabular}

Where $\boldsymbol{H p}$ and $\boldsymbol{L p}$ are highest and lowest prices in the last $\mathbf{p}$ days, respectively and $\boldsymbol{C}_{\boldsymbol{t}}$ is the current price of the day under consideration. AG is Average Gain and $\mathbf{A L}$ is Average Loss.

\subsection{Related Work}

To enhance the predictability of the daily stock price trends, Yuzheng Zhai et al. [11] presented a system based on SVM algorithm that combines the technical indicators and related news releases. For each trading day, seven technical indicators are computed from the prices in the past five days. Two groups of news releases are used. Two class categories, indicating the higher or equal price and lower price than close price, are taken up for indicating next day's price movement. The system achieved higher accuracy than achieved using single source i.e. news or technical indicators.

De Souza et al. [20] investigated the profitability of technical analysis as applied to the stock markets. Authors searched for evidence that fundamental analysis and technical analysis complement each other. An automated trading system is developed to simulate transactions in this portfolio using technical analysis techniques. The average results shown that the system exceeded the value invested. The sample portfolio from Russia and India showed very strong returns. The work utilized two types of moving average: SMA, EMA over varying number of days.

Pang et al. [21] proposed the deep LSTM with embedded layer (ELSTM) and the LSTM-NN network with automatic encoder to forecast the stock as traditional NN algorithms may incorrectly predict the stock market. The accuracy achieved with LSTM -NN with embedded layer is better. The maximum accuracy achieved is $57.2 \%$.

Chen et al. [22] proposes a novel technical analysis method for forecasting of stock market to increase the decision support quality and profitability of investors. The techniques utilize trend based classification, indicator selection, and stock market trading signal forecasting.

Hasan et al. [23] in their work utilized a number of ML techniques to predict the future stock prices. The ensemble is formed to combine the outcomes of different ML algorithms. To further enhance the prediction performance two popular and widely used technical indicators are pooled with the ML algorithms. To assess the proposed techniques the volume data and historical prices over the past 15 months of three prominent stocks are used. The algorithms predicts the 1-day, 1-week and 1-month-ahead prices of these stocks. The results signify that ensemble ML approach in combination with technical indicators often provide better results and less prediction error.

Xiong et al. [24] used economic variables and LSTM to predict the volatility of the S\&P index with the Google trend. Yu [25] applied the deep NN and LSTM to forecast the trading data of the Amazon stock. The highest prediction accuracy achieved is 54\%. It is mentioned that the effect of the deep NN was better than LSTM.

Zhang et al. [12] used the improved CNN and SVM to forecast the stock index and exchange rate in American,
European and Hongkong market. The result showed that the hybrid model had the highest accuracy [24].

Hiransha $M$ et al. [26], utilizes four deep learning architectures i.e. MLP, RNN, LSTM and CNN for predicting the stock price of a company based on the daywise historical closing prices of NSE of India and NYSE. The CNN performed much better even it is trained on NSE data and was able to predict for NYSE also. The results obtained were compared with linear ARIMA model.

Silva IND et al. [27] utilized RNN to forecast the prices of the three stocks. When used economic variables as input and the historical data, he found that the forecast price fitted the actual price better.

In stock market prediction, Neural Networks (NN) [19] has been shown as the most successful among ML models due to their ability to handle complex nonlinear systems. The predictive model for Stock market prediction is extremely complicated and $\mathrm{NN}$ gives it the ability to obtain higher accuracy. It is called Deep NN since we often get performance when adding extra layers.

There are two common deep learning architectures: the Recurrent Neural Networks (RNNs) and Convolutional Neural Networks (CNNs) [19] for predicting the stock price of a company based on the historical prices are available. Long Short Term Memory (LSTM) which is used in this work is a type of RNN with a significantly different architecture.

\section{PROPOSED WORK}

\subsection{Concept of Correlation-Tensor}

Deep Learning model characterizes numerical or categorical features as numbers. The indicator values are represented as simple vectors. The method has two primary drawbacks:

1. The vectors often acquire size which is equivalent to the size and number of indicators overwhelming the size of memory. Such large dimension vectors make computation inconvenient.

2. Also, the representation makes processing and context semantic analysis cumbersome.

Deep Neural Network (DNN) operates on numbers where every neuron performs addition and multiplication operations on inputs and weights. The proposed model

suggests the input to the model through correlation tensors. These tensors bridge input data with our model. Tensors enable to transform diverse information in rich STIs into formats that the DNN can use, allowing easy and fast experiment. Also, the time series data is not directly feed into the model, instead its value is broken into different categories based on ranges of adaptive STIs. The phenomenon of transforming feature vectors to tensors is shown in Figure 1. The important TIs along with range and decision is depicted in Table 3.

\section{Tech_indicator(1) \\ Tech_indicator(2) \\ Tech_indicator(3) \\ Tech_indicator(4)}

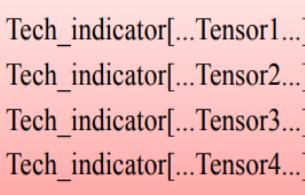

Fig. 1. Tensor Transformation Phenomenon

Published By:

Blue Eyes Intelligence Engineering \& Sciences Publication 
Table 3: Important Technical Indicators along with range and decision

\begin{tabular}{|c|c|c|c|c|}
\hline & \multicolumn{2}{|c|}{ RANGE } & \multicolumn{2}{|c|}{$\begin{array}{c}\text { PREDICTIO } \\
\mathbf{N} \\
\end{array}$} \\
\hline $\begin{array}{l}\text { TECHNICAL } \\
\text { INDICATOR }\end{array}$ & LOWER & UPPER & SELL & BUY \\
\hline Williams \%R & 0 & -100 & $\begin{array}{l}0 \text { TO- } \\
20\end{array}$ & $\begin{array}{l}-80 \\
\text { TO - } \\
100 \\
\end{array}$ \\
\hline $\begin{array}{l}\text { Moving } \\
\text { Average } \\
\text { Convergence/ } \\
\text { Divergence } \\
\text { Oscillator } \\
\text { (MACD) } \\
\end{array}$ & \multicolumn{4}{|c|}{$\begin{array}{l}\text { MACD does not have any upper or } \\
\text { lower limits }\end{array}$} \\
\hline $\begin{array}{l}\text { Money Flow } \\
\text { Index (MFI) }\end{array}$ & 0 & 100 & $\begin{array}{c}80 \text { to } \\
100 \\
\end{array}$ & $\begin{array}{c}20 \text { to } \\
0\end{array}$ \\
\hline $\begin{array}{l}\text { Commodity } \\
\text { Channel Index } \\
(\mathrm{CCI})\end{array}$ & $-\infty$ & $\infty$ & $\begin{array}{c}\text { above } \\
100\end{array}$ & $\begin{array}{c}\text { belo } \\
\mathrm{W}- \\
100 \\
\end{array}$ \\
\hline $\begin{array}{l}\text { Chaikin } \\
\text { Money Flow } \\
\text { (CMF) } \\
\end{array}$ & -1 & 1 & $<0$ & $>0$ \\
\hline $\begin{array}{l}\text { Average } \\
\text { Directional } \\
\text { Index (ADX) }\end{array}$ & 0 & 100 & $<20$ & $>40$ \\
\hline
\end{tabular}

At the beginning the correlation vectors derived using STIs. The Pearson correlation coefficient [28] is employed to derive the correlation among quantitative attributes. It is most suitable method as a precise numerical value of the degree of linear relationship between two variables $\mathrm{X}$ and $\mathrm{Y}$ is obtained and $\mathrm{NN}$ are based on the relationship ( $Y=$ $\left.W^{T} X+b\right)$. Then the derived correlation vectors are reduced and transformed as range of tensors with correlated or adaptive STIs. Tensors can be represented even in low dimension space than vectors. Finally, based on method of embedded correlation-tensor layer, a deep LSTM neural network model (OPTIMAL LSTM) is proposed.

\section{2 'Optimal Long Short Term Memory' Approach for} Stock Trends forecasting using STIs

LSTM is adaptive to resolve the problem of vanishing gradients especially in or long term forecasting problems therefore LSTMs are said as 'go-to' network for RNN. Originally LSTM was developed to extend the RNN memory state to enable it to deal with longer input sequences [28] like stock data. The RNN has only a standard tanh layer in each repeating module while LSTM has four layers as shown in Figure 2.

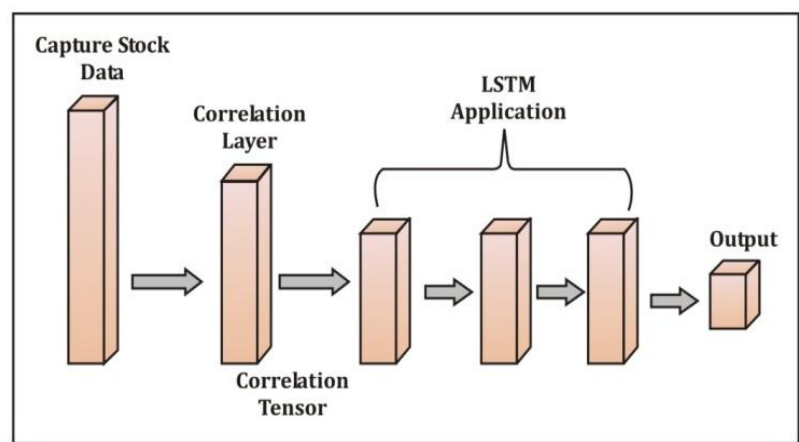

Fig. 2. Network Composition Diagram

\subsubsection{Optimal - LSTM Algorithm}

In the network composition above, the core module is LSTM. The node architecture with 4 transition phases is represented in Figure 3.

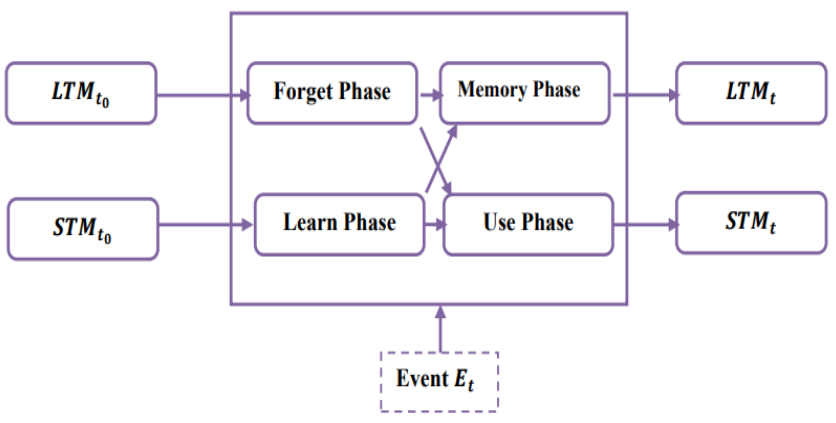

Fig. 3. Architecture of Optimal LSTM node

$E_{t}:$ Input Event.
$S T M_{t_{\mathrm{n}}}:$ Short Term Memory from previous time.
$W_{n}, W_{n}, W_{f}$ and $W_{u}:$ Linear Activation Functions applied to NN.
$N_{t}:$ Memory output at time t.
$i_{t}:$ ignore factor.
$L T M_{t_{\mathrm{n}}}:$ Long Term Memory from previous time.
$f_{t}:$ forget factor.
$S T M_{t}:$ New short term output of EDLA node
$L T M_{t}:$ New long term output of EDLA node.
tanh : ReLU.
$\sigma:$ Sigmoid Function.
$U_{t} \& V_{t}$ are calculations with Use Phase inputs.
$b_{n}, b_{i}, b_{f}, b_{u}$ and $b_{v}$ are bias.

Optimal - LSTM Algorithm 


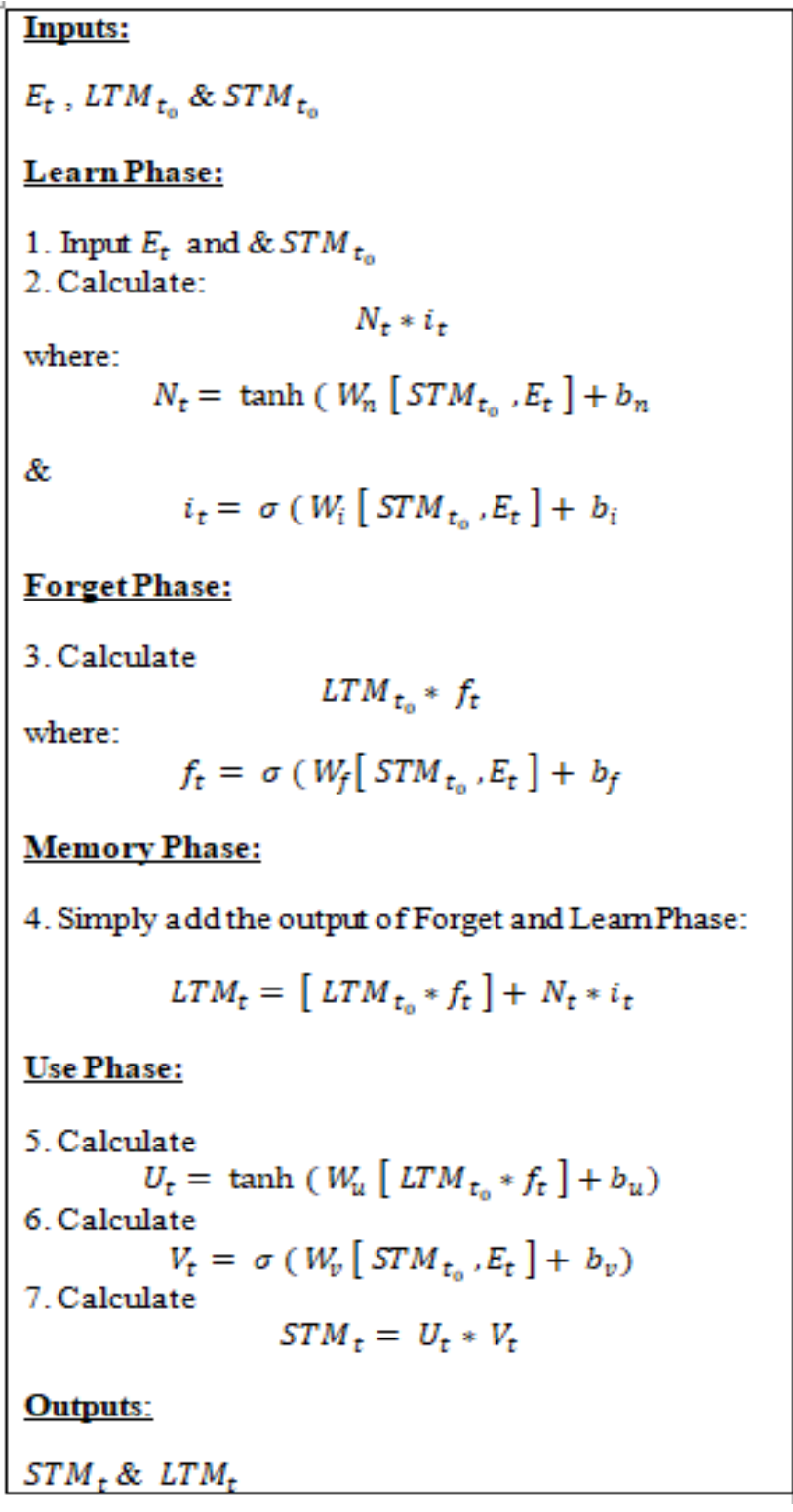

\subsubsection{Construction of Proposed Framework}

The proposed framework is presented in Figure 4.

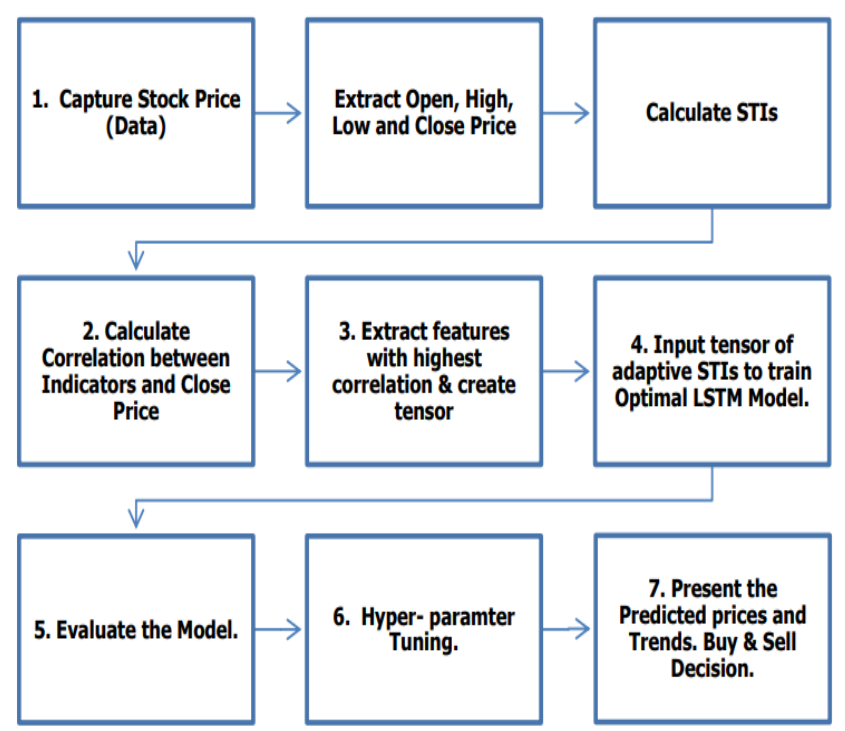

Fig. 4: Proposed Framework for Stock Trends Forecasting
The stock data is captured through APIs. The Open, High, Low and Close (OHLC) prices are extracted from the dataset. We will build the new input features, which are known as STIs, by applying technical analysis. Later we derive the correlation between STIs and Close Price. The input to the phase 4 depends on the correlation tensor derived in phase 3 . The highly correlated features are extracted along with close price are supplied to Optimal LSTM model in phase 4. The 3-day, 10-day and 30-day Moving Averages performed much better in this work.

The Optimal LSTM cell keeps track of not just of Memory but of long and short term memory as well which comes in and out of cells.

The input of each Optimal LSTM node is pretentious by previous layer and also receives the effect of the same layer processing. The linear activation function is used for performing regression prediction. For trends prediction the function is logistic. When predicting the accuracy, the stock's close price is divided into two classes' price-rise (1) and prise-fall (0). The indication of price-rise (1) specifies the Buy Signal at the end of the day, while the prise-down (0) indicates the Sell of stock. Finally the model presents the strategic trends and prediction of stock prices. The model is evaluated on the basis of Prediction Accuracy (y) and Mean Squared Error (MSE) as represented in section 4.4.

\section{EXPERIMENT SETUP, RESULTS AND ANALYSIS}

\subsection{About Dataset}

The data of 3 financial organizations (Banks) listed in National Stock Exchange (NSE) - India, is captured through Yahoo Finance. The banks chosen either belong to nationalized, public or private sectors. The data set encompassed the trading days of 2 years i.e. from $16^{\text {th }}$ Nov, 2016 to $15^{\text {th }}$ Nov, 2018.

\subsection{Experiment Environment Setup}

The experiments are performed on system with Intel Core i3 (2.0 GHz.) processor and 8GB RAM, running Microsoft Windows - 10 (64-bit) operating system. The Environment and Libraries used in work are presented in Table 4 . The model is developed in Python language (version 3.7.0 and 3.6.6) utilizing Keras as the high-level API developed for Google's TensorFlow.

Table 4: Environments and Libraries

\begin{tabular}{ll}
\multicolumn{1}{c}{$\begin{array}{c}\text { Environments and } \\
\text { Libraries }\end{array}$} & \multicolumn{1}{c}{ Version / Configuration } \\
\hline Anaconda Navigator & v_5.3.1; x86_64 bit \\
Jupyter Notebook & v_5.7.2; 64 bit \\
SciPy & 1.1 .0 \\
NumPy & 1.15 .4 \\
Scikit-Learn & 0.20 .2 \\
TensorFlow & 1.12 .0 \\
\hline
\end{tabular}


Stock Price Prediction using Technical Indicators: A Predictive Model using Optimal Deep Learning

\begin{tabular}{ll}
\hline Keras & $\mathbf{2 . 2 . 4}$ \\
\hline Pandas & 0.23 .4 \\
Seaborn and Matplotlib & 0.9 .0 and 3.0.2 respectively \\
\hline
\end{tabular}

\subsection{Experiments conducted}

We conducted several experiments to

- To study the correlation between popular technical indicators and close price.

- $\quad$ To evaluate the performance of proposed work against state-of-the-art methods.

The proposed approach can be used for trend based analysis as it helps trader to decide whether to hold shares for long or short term. Also, an additional field is added to training dataset as indicator for providing Buy and Sell signal at the end of each day. Price-rise (1) or Price-fall (0) indicates Buy and Sell signal respectively.

\subsection{Classifier Results, Performance Evaluation and Discussion}

The major outcomes of model are obtained at step 2, 3, 4 and 7. Figure 5, reveals the correlation cluster-map of HDFC bank which is obtained after step 2. The correlation map represents the correlation value between the Price-rise and STIs that are calculated.

In this experimental study most adaptive STIs are extracted and suitably pre-processed. The adaptive TAs calculated for three banks are presented in Table 5 .

The final outputs of experiment are obtained at stage 7 . The types of outputs are mentioned below:

1. Price-rise or Price-fall decision for intraday trading:

2. Long and short term trend analysis for weekly or monthly trading.

\subsubsection{Correlation Matrix}

Figure 5 showing correlation map between OHLC and STIs for HDFC bank. This work involves study of several indicators, out of which Moving Averages performed well. Table 5 showing correlation values between close price of 3 stocks and MAs, which is output of step 2.

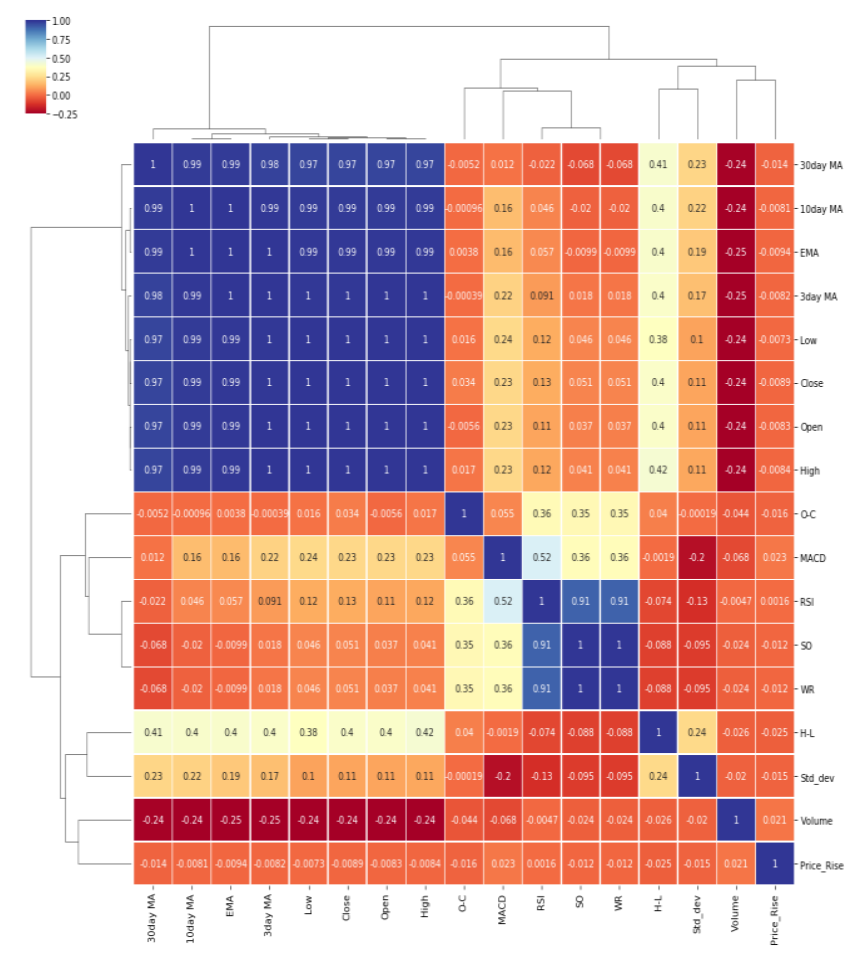

Fig. 5: Correlation Map for HDFC Stock

Table 5: Correlation Values between MAs and Close Price

\begin{tabular}{|c|c|c|c|c|}
\hline & & \multicolumn{3}{|c|}{ Correlation Values } \\
\hline & & $\begin{array}{c}0 \text { day } \\
\text { MA }\end{array}$ & $\begin{array}{c}10 \text { day } \\
\text { MA }\end{array}$ & $\begin{array}{c}3 \text { day } \\
\text { MA }\end{array}$ \\
\hline \multirow{3}{*}{ 䓪 } & HDFC & 0.98 & 0.99 & 1.00 \\
\hline & $\begin{array}{l}\text { Yes } \\
\text { Bank }\end{array}$ & 0.93 & 0.97 & 0.99 \\
\hline & SBI & 0.66 & 0.88 & 0.95 \\
\hline
\end{tabular}

\subsubsection{Prediction Accuracy of Optimal LSTM:}

When the training batch size voted is 30 and classifier is build with Optimal LSTM on varying number of epochs. The Model built and evaluated on the basis of prediction accuracy and MSE. The decision value 1 stands for Pricerise for next day and 0 stands for Price-fall on next day. We can say that 1 indicates Buy and 0 indicates Sell the stock at the end of day.

$$
y=\left\{\begin{array}{lr}
1, & \left(y_{i+1}-y_{i}\right)>0 \\
0 & \text { otherwise }
\end{array}\right\}
$$

The percentage accuracy achieved over different values of epochs is depicted in Table 6. The results are mentioned up to 500 epochs as the model started over-fitting the data beyond this.

Table 6: Accuracy of Optimal LSTM with 3 Datasets

\begin{tabular}{lccc}
\hline \multicolumn{3}{c}{ Accuracy (\%) } \\
\hline Epochs & YES Bank & HDFC & SBI \\
$\mathbf{1 0 0}$ & 56.13 & 61.04 & 55.59 \\
$\mathbf{2 0 0}$ & 57.98 & 62.58 & 58.39 \\
$\mathbf{3 0 0}$ & 48.77 & 64.11 & 53.42 \\
$\mathbf{4 0 0}$ & 58.9 & 64.42 & 61.49 \\
$\mathbf{5 0 0}$ & 59.51 & 65.64 & 60.87
\end{tabular}

The graph representation of accuracy achieved using different number of epochs is shown in Figure 6.

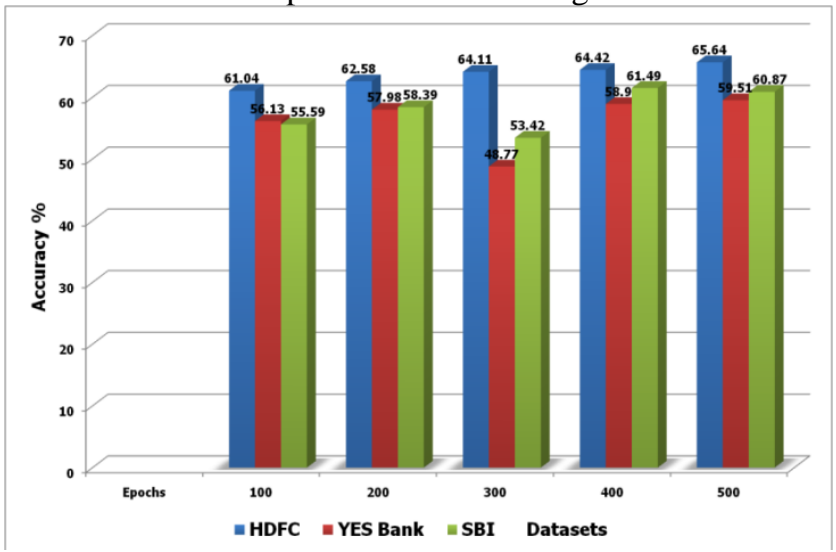

Fig. 6. Optimal LSTM Accuracy (\%) Comparison across 3 Bank Stock Datasets

\subsubsection{Mean Squared Error (MSE):}

Another metric evaluated is MSE. It measures the average of average of squares of the errors in a set of predictions.

$$
M S E=\frac{1}{n} \sum_{k=1}^{n}\left(\mathrm{y}_{\mathrm{k}}-\hat{\mathrm{y}}_{\mathrm{k}}\right) 2
$$

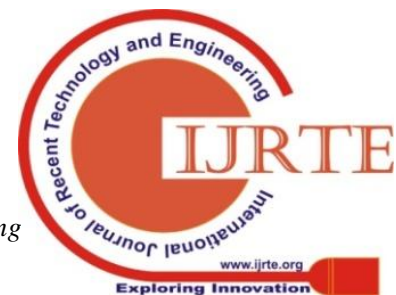


where $\hat{y}_{\mathrm{k}}$ and $\mathrm{y}_{\mathrm{k}}$ are Predicted and Actual outputs respectively.

The MSE comparison of MLP, ELSTM and Optimal LSTM is shown in Figure 7. The average value of MSE across 3 datasets is chosen for comparison.

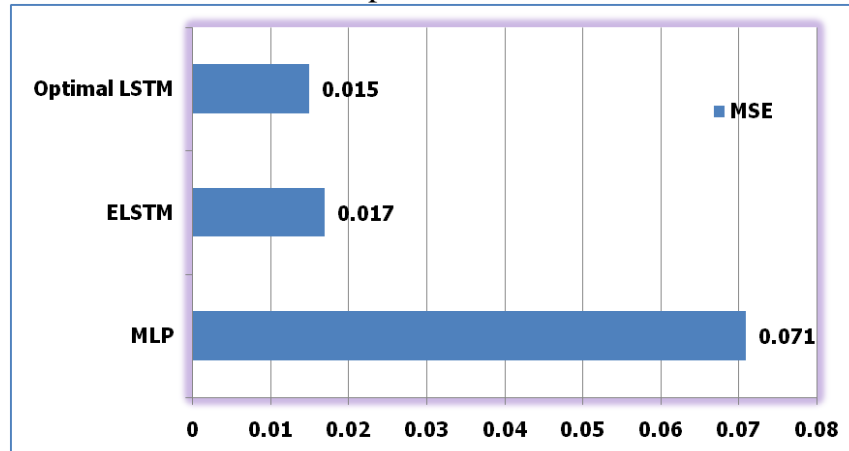

Fig. 7. MSE Comparison for MLP, ELSTM and Optimal LSTM

\subsubsection{Stock Trend Predictions}

The trends obtained for all the three banks are represented in Fig. 8 to Fig. 10. The trends primarily predicted to take long and short positions with stock. The graphs are showing the comparison of actual v/s predicted price trends for 141 days, i.e. for 30 percent of 2 years data.

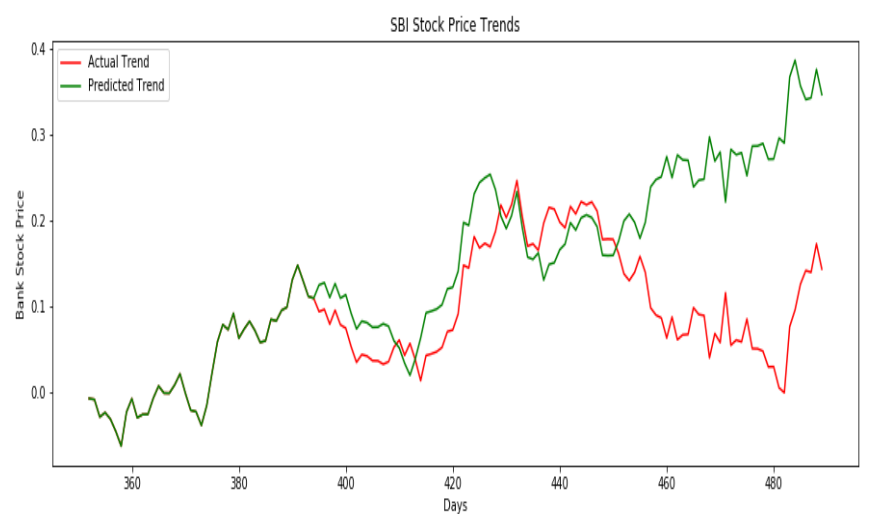

Fig. 8. SBI Stock Trend Actual v/s Predicted

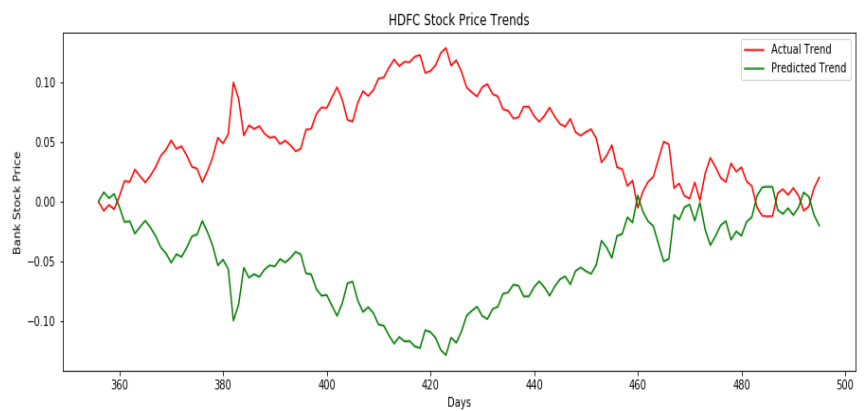

Fig. 9. HDFC Stock Trend Actual v/s Predicted

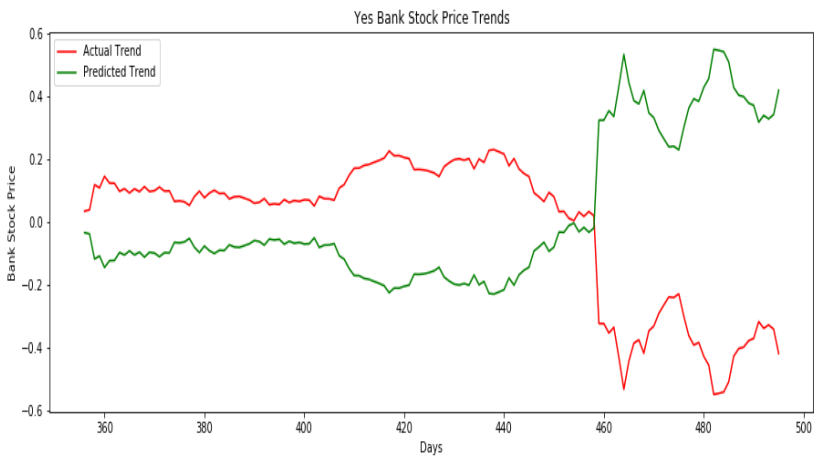

Fig. 10. Yes Bank Stock Trend Actual v/s Predicted

\subsubsection{Performance Evaluation}

The accuracy of proposed model is also compared with 2 benchmark ML classifiers: Support Vector Machine (SVM), Logistic Regression (LR) and one deep learning model: ELSTM [21]. The results are presented in Table 7 whose graph representation is shown in Figure 11.

Table 7: Accuracy Comparison of SVM, LR and ELSTM with Optimal LSTM

\begin{tabular}{lcccc}
\hline \multicolumn{5}{c}{ Accuracy (\%) } \\
\hline HDFC & 51.06 & 52.48 & 57.2 & 63.59 \\
YES & 49.64 & 50.35 & 52.6 & 56.25 \\
BANK & & & & Optimal \\
SBI & 55.39 & 56.11 & 56.8 & 57.95 \\
\hline
\end{tabular}

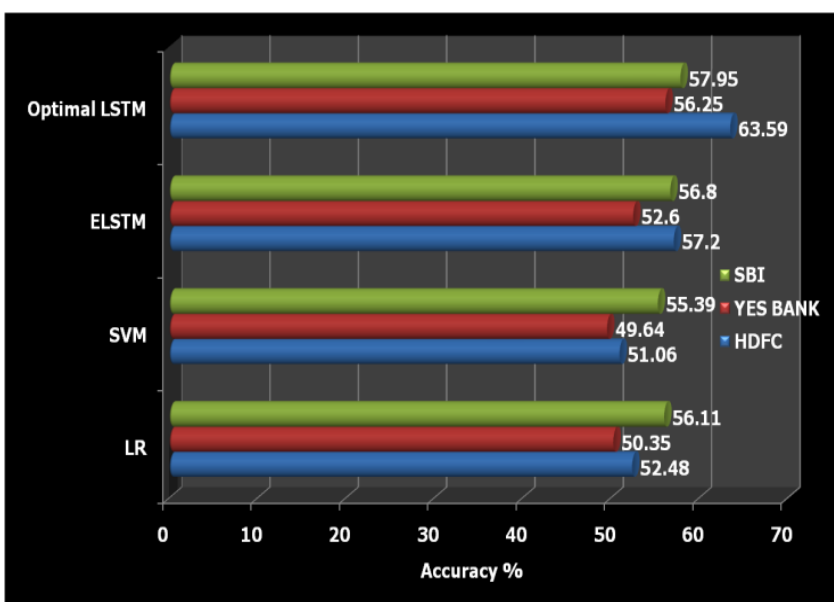

Fig. 11. Accuracy Comparison of SVM, LR and ELSTM with Optimal LSTM

\subsubsection{Discussion}

It is clearly depicted from results that the application of Optimal LSTM along with correlation tensor of adaptive STIs improves prediction performance. The highest accuracy and mean accuracy achieved is $65.64 \%$ and $59.25 \%$ respectively, which is much higher than SVM, LR and a deep learning approach (ELSTM) [21]. The proposed framework with Optimal LSTM is capable of exploring the correlative STIs. As an outcome the model predicts the long and short term market trends of any stock. The time period in which the predicted trends almost follow actual trend is considered better for future investments. However, the uncertain trends justify that there might be risk involved with particular stock for particular time periods. The outcomes of model demonstrate that the investor will definitely fetch more profit by taking efficient decision of buying and selling the stock. The model explicitly achieved uppermost prediction accuracy without over-fitting. The number of epochs is chosen to avoid over-fitting. The mean accuracy of Classifier achieved is $59.25 \%$, over number of datasets. The MSE is least across all classifiers including Multi-Layer Perceptron. Further experiments reveal that the STIs such as MA and MACD are highly correlated with close price.

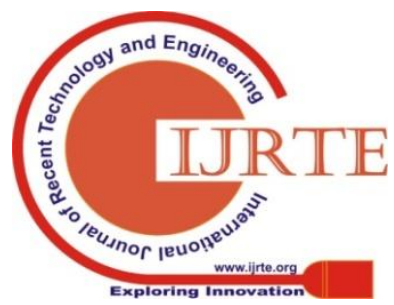




\section{Stock Price Prediction using Technical Indicators: A Predictive Model using Optimal Deep Learning}

The model built is market independent and is implemented in Python using powerful ML and NN libraries: Google TensorFlow and Keras. Performance evaluation and comparison of has shown pre-eminence of proposed work.

\section{CONCLUSION AND FUTURE DIRECTIONS}

We inspected the efficiency of applying technical analysis to the stock prices. We analyzed whether investors could manage more profits than suggested by the recent research of Pang X. et al. (2018). We demonstrated the concept of deriving adaptive STIs and passing them as correlation tensor. The tensor is then supplied to the model. Finally the model presents a well-organized approach that aids traders for long/short as well as daily trading and to earn profit. Optimal LSTM presents decision based indicator (Price-rise (1) or Price-fall (0)) as well as trend based analysis. The proposed Optimal Deep Learning Approach (Optimal LSTM) is a market independent approach as we are discovering the potential indicators existing in the data and applying LSTM dynamics of deep learning rather than fixing data or model.

This work opens several research confronts to get more insights on stock trends forecasting. In future the research work can be extended by applying more STIs and can be evaluated against several ML and deep learning approaches. The proposed model can be further evaluated and optimized for stock indices. The proposed deep learning algorithm can also be further enhanced to optimize the performance. To support investors, the proposed model can be further integrated into an automated system for trading on specific stocks.

\section{REFERENCES}

1. 1 Sepp Hochreiter, Jürgen Schmidhuber, Long Short-Term Memory, Journal Neural Computation, Volume 9 Issue 8, November 15, 1997, Pages 1735-1780

2. 2 Stelios A. Mitilineos; Panayiotis G. Artikis, "Forecasting of future stock prices using neural networks and genetic algorithms", International Journal of Decision Sciences, Risk and Management, 2017 Vol.7 No.1/2, pp.2 - 25

3. 3 F. B. Oriani and G. P. Coelho, "Evaluating the impact of technical indicators on stock forecasting," 2016 IEEE Symposium Series on Computational Intelligence (SSCI), Athens, 2016, pp. 1-8.

4. 4 G. Zhang, X. Zhang, and H. Feng, "Forecasting financial time series using a methodology based on autoregressive integrated moving average and taylor expansion," Expert Systems, vol. 33, no. 5, pp. 501516, 2016.

5. 5 M. Valipour, M. E. Banihabib, and S. M. R. Behbahani, "Comparison of the ARMA, ARIMA, and the autoregressive artificial neural network models in forecasting the monthly inflow of Dez dam reservoir," Journal of Hydrology, vol. 476, pp. 433-441, jan 2013.

6. 6 Sundaravelpandian Singaravel, Johan Suykens, Philipp Geyer, "Deep-learning neural-network architectures and methods: Using component-based models in building-design energy prediction", Advanced Engineering Informatics, Volume 38, 2018, Pages 81-90.

7. 7 V.N. Gudivada, Chapter 1 - Cognitive Computing: Concepts, Architectures, Systems, and Applications, Editor(s): Venkat N. Gudivada, Vijay V. Raghavan, Venu Govindaraju, C.R. Rao, Handbook of Statistics, Elsevier, Volume 35, 2016, Pages 3-38.

8. 8 Edward Gately, Neural Networks for Financial Forecasting, John Wiley \& Sons, Inc., New York, NY, 1995

9. 9 F. A. de Oliveira, C. N. Nobre, and L. E. Z'arate, “Applying artificial neural networks to prediction of stock price and improvement of the directional prediction index - case study of PETR4, Petrobras, Brazil,"

10. 10 Expert Systems with Applications, vol. 40, no. 18, pp. 75967606, dec 2013

11. 11 Zhai Y., Hsu A., Halgamuge S.K. (2007) Combining News and Technical Indicators in Daily Stock Price Trends Prediction. In: Liu D., Fei S., Hou Z., Zhang H., Sun C. (eds) Advances in Neural Networks ISNN 2007. ISNN 2007. Lecture Notes in Computer Science, vol 4493. Springer, Berlin, Heidelberg
12. 12 Zhang G-Y (2016) Application of improved convolution neural network in financial forecasting. Zhengzhou University, Henan

13. 13 Yann LeCun, Yoshua Bengio, and Geoffrey Hinton. Deep learning. Nature, 521(7553):436-444, 2015

14. 14 G. Batres-Estrada, "Deep learning for multivariate financial time series," ser. Technical Report, Stockholm, May 2015.

15. 15 E. Chong, C. Han, and F. C. Park, "Deep learning networks for stock market analysis and prediction: Methodology, data representations, and case studies," Expert Systems with Applications, vol. 83, pp. 187-205, 2017.

16. s16 Jabbarzadeh, Armin, et al. "A Multiple-Criteria Approach for Forecasting Stock Price Direction: Nonlinear Probability Models with Application in S\&P 500 Index." International Journal of Applied Engineering Research 11.6 (2016): 3870-3878.

17. 17 Jan Ivar Larsen. Predicting stock prices using technical analysis and machine learning. NTNU, 2010.

18. 18] F. B. Oriani and G. P. Coelho, "Evaluating the impact of technical indicators on stock forecasting," 2016 IEEE Symposium Series on Computational Intelligence (SSCI), Athens, 2016, pp. 1-8.

19. 19 M. Nielsen, Neural Networks and Deep Learning (Determination Press, 2015).

20. 20 De Souza, M.J.S., Ramos, D.G.F., Pena, M.G. et al. Financ Innov (2018) 4: 3. https://doi.org/10.1186/s40854-018-0087-z

21. 21 Pang, X., Zhou, Y., Wang, P. et al. J Supercomput (2018) https://doi.org/10.1007/s11227-017-2228-y

22. 22 Chen, YJ., Chen, YM., Tsao, ST. et al. Soft Comput (2018) 22: 1295. https://doi.org/10.1007/s00500-016-2417-2

23. 23 Hasan S.S., Rahman R., Mannan N., Khan H., Moni J.N., Rahman R.M. (2017) Improved Stock Price Prediction by Integrating Data Mining Algorithms and Technical Indicators: A Case Study on Dshaka Stock Exchange. In: Nguyen N., Papadopoulos G., Jędrzejowicz P., Trawiński B., Vossen G. (eds) Computational Collective Intelligence. ICCCI 2017. Lecture Notes in Computer Science, vol 10448. Springer, Cham

24. 24 Xiong R, Nichols EP, Shen Y (2015) Deep learning stock volatility with google domestic trends. Preprint arXiv:1512.04916

25. 25 Yu F-F (2016) Based on the neural network of stock forecast [D]. Harbin Institute of Technology, Harbin

26. 26Hiransha M, Gopalakrishnan E.A., Vijay Krishna Menon, Soman K.P., NSE Stock Market Prediction Using Deep-Learning Models, Procedia Computer Science, Volume 132, 2018, Pages 1351-1362, ISSN 1877-0509, https://doi.org/10.1016/j.procs.2018.05.050.

27. 27 Silva IND, Spatti DH, Flauzino RA et al (2017) Forecast of stock market trends using recurrent networks. In: da Silva IN, Spatti DH, Flauzino RA, Liboni LHB, Alves SFR (eds) Artificial Neura Networks. Springer International Publishing, pp 221-227

28. 28Laura D. Goodwin \& Nancy L. Leech (2006) Understanding Correlation: Factors That Affect the Size of $r$, The Journal of Experimental Education, 74:3, 249-266, DOI: 10.3200/ JEXE.74.3.249-266.

\section{AUTHORS PROFILE}

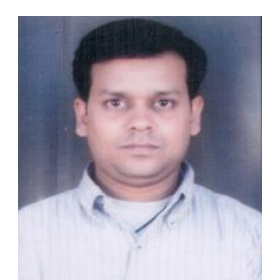

Author 1 : Manish Agrawal, He is presently pursuing $\mathrm{PhD}$ in Computer Science and Engineering. He has received his Bachelor's degree and M. Tech degree in Computer Science \& Engineering from RGPV University, Bhopal. He is a Life Member of Computer Society of India. His research interest includes Image Mining, Network Security, Artificial Intelligence and Deep Learning. His work experience includes 15 years in teaching computer science and engineering and 3 years as software engineer in Ray Solutions, Bhopal. At present he is working as software engineer in Ray Solutions, Bhopal. He is guided 2 M.Tech Students.

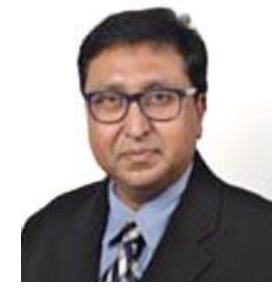

Author 2 : Dr. Asif Ullah Khan, is renowned educationist and administrator. He did his doctorate in Computer Science and Engineering from RGPV, Bhopal. He is the Gold Medalist in M.Tech from Rajiv Gandhi Technological University and got the B.E. degree from Samrat Ashok Technological Institute, Vidisha. He is having more than 25 years of experience in academics. 
$\mathrm{He}$ is associated with various Academic, Technical and Industrial collaborative activities for the students, and also associated intensively in research activities He was awarded the prestigious Bharat Shiksha Ratan Award in August,2013.He has visited and delivered expert lectures in Dubai, Malaysia, Thailand ,Singapore, France, Germany, Italy and Switzerland. He is a senior member of Institute of Electrical and Electronics Engineers .He is also a senior member of International Association of Computer Science and Information Technology. Currently he is working as Professor in Department of Computer Science \& Engineering, Technocrats Institute of Technology (E), Bhopal.

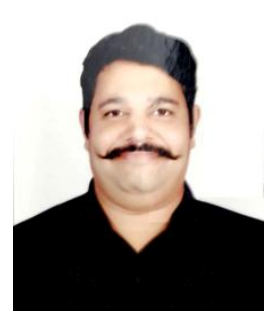

Author 3 : Dr. Piyush Kumar Shukla, received his Bachelor's degree in Electronics \& Communication Engineering, LNCT, Bhopal in 2001, M.Tech (Computer Science \& Engineering) in 2005 from SATI, Vidisha and Ph.D. (Computer Science \& Engineering) in 2013 from RGPV, Bhopal. M.P. India. He is a Member of ISTE (Life Member), IACSIT, IAENG Senior Member IEEE. Currently he is working as an Assistant Professor (Grade 8000/-) in Department of Computer Science \& Engineering, UIT-RGPV Bhopal. He is also I/C of PG Program (Dual Degree Integrated PGPrograms) in DoCSE, UIT, RGPV, Bhopal, Madhya Pradesh. He has published more than 60 Research Papers in various International \& National Journals \& Conferences, including 04 Papers in SCIE Journals \& More than 10 papers in Scopus Journals. He has also published an Indian patent. 